\title{
Estradiol promotes EMT in endometriosis via MALAT1/ miR200s sponge function
}

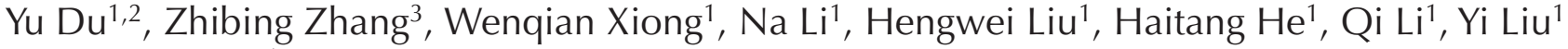 \\ and Ling Zhang ${ }^{1}$ \\ ${ }^{1}$ Department of Obstetrics and Gynecology, Union Hospital, Tongji Medical College, Huazhong University of \\ Science and Technology, Wuhan 430022, China, ${ }^{2}$ Department of Obstetrics and Gynecology, Tongji Hospital, Tongji \\ Medical College, Huazhong University of Science and Technology, Wuhan 430030, China and ${ }^{3}$ Department of \\ Physiology and Obstetrics and Gynecology, Wayne State University, Detroit, Michigan, USA
}

Correspondence should be addressed to Y Liu or L Zhang; Email: liqun1994@hust.edu.cn or zhanglingxh@hust.edu.cn

\begin{abstract}
Endometriosis is an estrogen-dependent benign gynecological disease that shares some common features of malignancy. Epithelialmesenchymal transition (EMT) has been recognized as a core mechanism of endometriosis. MALAT1 is widely known as EMT promoter, while miR200 family members (miR200s) are considered as EMT inhibitors. Previous studies have reported that MALAT1 upregulation and miR200s downregulation are observed in endometriosis. MiR200c has been regarded as the strongest member of miR200s to interact with MALAT1. However, whether MALAT1/miR200c regulates EMT remains largely unclear. In this study, the roles of miR200s and MALAT1 in ectopic endometrium were investigated. Additionally, the effects of $E_{2}$ on EMT and MALAT1/ miR200s were examined in both EECs and Ishikawa cells. Notably, $E_{2}$ could upregulate MALAT1 and downregulate miR200s expression levels and induce EMT in EECs and Ishikawa cells. PHTPP, an ERß antagonist, could reverse the effect of $E_{2}$. Overexpression of miR200c and knockdown of MALAT1 significantly inhibited $E_{2}$-mediated EMT, suggesting that both miR200c and MALAT1 are involved in the $E_{2}$-induced EMT process in endometriosis. In addition, a reciprocal inhibition was found between miR200s and MALAT1. Therefore, the role of MALAT1/miR200c in EMT is influenced by the presence of estrogen during endometriosis development.

Reproduction (2019) 157 179-188
\end{abstract}

\section{Introduction}

Endometriosis is a common benign gynecological disease characterized by the presence of endometrial glands and stroma outside the uterine cavity (Giudice \& Kao 2004). Its main symptoms include chronic pelvic pain and infertility, which can seriously impair patients' quality of life (Crosignani et al. 2006). Although endometriosis is a benign disease, its characteristics of metastasis and implantation are similar to tumors. Epithelial-mesenchymal transition (EMT) is characterized by the loss of epithelial phenotype and the acquisition of mesenchymal features (Yang \& Weinberg 2008). The increased expression levels of $\mathrm{N}$-cadherin and Vimentin as well as reduced E-cadherin expression are the most influential biomarkers of EMT. Besides, EMT-associated transcription factors, such as Zeb1, Zeb2, Snail, Slug and Twist, can promote EMT by regulating EMT markers (Yang \& Weinberg 2008, Vandewalle et al. 2009). Previous studies have shown that EMT is associated with the formation of endometriotic lesions (Proestling et al. 2015, Xiong et al. 2016). However, the molecular mechanisms underlying the role of EMT in endometriosis remain largely unknown.

The miR200 family (miR200s), including five members (miR200a, miR200b, miR200c, miR141 and miR429), are well recognized as EMT inhibitors, and served as the molecular signatures of tumor suppression (Feng et al. 2014). MiR200s mainly inhibit Zeb1 and Zeb2, preventing these transcription factors from triggering EMT. Compared to healthy endometrium, miR200s expression levels have been downregulated in the ectopic endometrium of endometriosis patients (Hawkins et al. 2011). Therefore, miR200s may play an important role in EMT during endometriosis development. Long non-coding RNAs (IncRNAs) often act as competing endogenous RNAs (ceRNAs) by functioning as miRNA sponges (Dhamija \& Diederichs 2016). LncRNA MALAT1, upregulated in several cancers, may promote the development and metastasis of tumors by promoting EMT (Xiao et al. 2015, Yoshimoto et al. 2016). A recent study demonstrated that expression of MALAT1 in ectopic endometrial specimens is negatively correlated with that of miR200c, while MALAT1 knockdown 
increased the level of miR200c in endometrial stromal cells (Liang et al. 2017). The finding suggested that MALAT1/miR200c may participate in the development of endometriosis by affecting EMT. However, the molecular mechanism underlying the effects of MALAT1/miR200c has not been fully elucidated.

The etiology and pathogenesis of endometriosis remain controversial and poorly understood, but the theory of Sampson is widely accepted (Sampson 1927). Endometrial tissues flow from the uterus the fallopian tubes during retrograde menstruation, followed by migration and implantation in the secondary sites, which can lead to endometriosis. High levels of localized estrogen may play a key role in the development and progression of endometriosis (Kitawaki et al. 2002). Therefore, endometriosis has been viewed as an estrogendependent disorder. $17 \beta$-Estradiol $\left(E_{2}\right)$, the most potent estrogen synthesized in vivo, mainly operates through estrogen receptor $\alpha(E R \alpha)$ and estrogen receptor $\beta(E R \beta)$ (Matthews \& Gustafsson 2003, Huhtinen et al. 2012). Estrogen has been demonstrated to induce EMT in cancer cells through several distinct mechanisms (Park et al. 2008, 2016). Furthermore, estrogen participated in the induction of EMT in adenomyosis (Chen et al. 2010).

Considering the importance of $E_{2}$ in promoting EMT in endometriosis, we hypothesize that $E_{2}$ may induce EMT in endometriosis by altering the expression levels of MALAT1 and miR200s. In the present study, bioinformatics analysis was used to predict the upstream transcription factors of MALAT1. The discovery of estrogen response element (ERE) on MALAT1 promoter region indicated the underlying relationship between estrogen and MALAT1. A high level of localized $E_{2}$ in endometriosis may enhance MALAT1 expression and contribute to the reduction of miR200s. In addition, the inhibitory effects of miR200s on Zeb1 and Zeb2 expressions were counteracted, resulting in the downregulation of E-cadherin during EMT process in endometriosis.

\section{Materials and methods \\ Ethical approval}

Ethical approval for this study was obtained from the Ethics Committee of Tongji Medical College, Huazhong University of Science and Technology (IORG No: IORG0003571). All tissue samples were obtained with full and informed patient consent.

\section{Patients and tissue collection}

Twenty ovarian chocolate cysts (ectopic endometriotium of endometriosis patients, mean age: $29.2 \pm 4.0$, stage III-IV) and 20 eutopic endometrium (mean age: $30.1 \pm 3.9$, stage III-IV) were obtained by biopsy undergoing laparoscopy in this study. Twenty specimens of normal endometrium (mean age: $31.4 \pm 4.6$ ) without endometriosis were collected by curettage for tubal infertility to compare with ectopic and eutopic groups (Table 1). Sixty-four specimens of normal endometrium were collected by curettage to culture human primary endometrial glandular epithelial cells (EECs). Patients were recruited from the Department of Obstetrics and Gynecology, Union Hospital, Tongji Medical College, Huazhong University of Science and Technology. All recruited women were premenopausal and without any hormonal treatments for at least 3 months. They all had regular menstrual cycles and the samples were collected during the proliferative phase to exclude the interference of progestin. The normal, ectopic and eutopic tissues for qRT-PCR were immediately washed with ice-cold PBS on ice and transferred into TRIzol reagent at $-80^{\circ} \mathrm{C}$. Endometrial tissues for primary cell culture were immediately stored in ice-cold PBS and the isolation primary cells was performed immediately.

\section{Cell culture and transfection}

Human primary EECs were isolated from normal fresh endometrial tissues. The tissues were washed in PBS and digested with collagenase II (0.1\%; Sigma-Aldrich). Then, the tissue pieces were filtered through a $150 \mu \mathrm{m}$ sieve to remove debris, and followed by a $38 \mu \mathrm{m}$ sieve to separate the EECs from stromal cells. EECs were resuspended in a 1:1 mixture of phenol red-free DMEM/F12 (Gibco) with $20 \%$ fetal bovine serum (FBS; Gibco), $100 \mathrm{U} / \mathrm{mL}$ penicillin and $100 \mu \mathrm{g} / \mathrm{mL}$ streptomycin (Beyotime). Ishikawa cells (Shanghai Fuxiang Biotechnology Co. Ltd., China) were cultured in phenol red-free 1640 (Gibco) with 10\% FBS and antibiotics. Both cell lines were maintained in a humidified $5 \% \mathrm{CO}_{2}$ incubator at $37^{\circ} \mathrm{C}$.

Two MALAT1-specific siRNAs, the miR200c mimic and the miR200c inhibitor, were synthesized by RiboBio (Guangzhou, China, si-MALAT1-1: No.siB170710110803; si-MALAT1-2: siB121112152859; si-NC: siN05815122147). They were respectively transfected into Ishikawa cells $\left(3 \times 10^{6}\right.$ per $\left.\mathrm{mL}\right)$ by using Lipofectamine 2000 (Invitrogen) according to the manufacturer's protocol. The sequences were listed as follows: has-miR200c-3p mimic: 5'-UAAUACUGCCGGGUAAUGAUGGA-3'; mimic Ncontrol: micrONTM miRNA mimic Ncontrol \#22; and has-miR200c-3p inhibitor: 5'-UAAUACUGCCGGGUAAUGAUGGA; Inhibitor Ncontrol: micrOFFTM miRNA inhibitor Ncontrol \#22.

\section{Hormone treatment}

$\mathrm{E}_{2}$ (Sigma-Aldrich, E-2758), ICI $182780(\mathrm{ICl})$, an estrogen receptor $(E R \alpha)$ antagonist (CAS 129453-61-8) and PHTPP, an estrogen receptor (ER $\beta$ ) antagonist (CAS 805239-56-9) were dissolved in DMSO. EECs and Ishikawa cells were treated with different concentrations $\left(0,10^{-12}, 10^{-10}, 10^{-8}\right.$ and $10^{-6} \mathrm{~mol} / \mathrm{L}$ ) of $\mathrm{E}_{2}$ and incubated at different time points $(0,24,48,72 \mathrm{~h})$. The cells were incubated with serumfree and phenol red-free DMEM/F12 or 1640 medium for $24 \mathrm{~h}$ before hormone treatment. For evaluating the effect of estrogen receptor antagonists, PHTPP was added to the cells $24 \mathrm{~h}$ before $\mathrm{E}_{2}$ treatment. All medium were replaced every $24 \mathrm{~h}$. 
Table 1 Clinical characteristics of patients.

\begin{tabular}{|c|c|c|c|c|}
\hline & \multicolumn{3}{|c|}{ Samples for RT-PCR } & \multirow{2}{*}{$\begin{array}{l}\text { Samples used for primary cell } \\
\text { cultures }\end{array}$} \\
\hline & Normal endometrium & Eutopic endometrium & Ectopic endometrium & \\
\hline Number of cases & 20 & 20 & 20 & 64 \\
\hline Age* $^{*}$ & $31.4 \pm 4.6$ & $29.2 \pm 4.0$ & $30.1 \pm 3.9$ & $29.9 \pm 4.1$ \\
\hline $\begin{array}{l}\text { Menstrual cycle phase } \\
\text { rASRM stage }\end{array}$ & Proliferative & Proliferative & Proliferative & Proliferative \\
\hline III & - & 8 & 11 & - \\
\hline IV & - & 12 & 9 & - \\
\hline
\end{tabular}

*Median (range). "Revised American Society for Reproductive Medicine classification (1997).

\section{$R N A$ extraction and quantitative real-time PCR (qRT-PCR)}

Total RNA, including miRNA, was extracted from frozen tissues and cultured cells with Qiagen miRNeasy Mini Kit (Qiagen). Isolated RNA was reversed-transcribed by PrimeScript RT Master Mix (Takara). QRT-PCR assay was prepared with SYBR Premix Ex Taq (Takara). The expression of MALAT1 and other mRNAs were normalized to GAPDH expression level. MiRNAs were reverse-transcribed and detected by qRT-PCR using RIBOBIOmiDETECT A Track miRNA qRT-PCR Starter Kit (RiboBio, Guangzhou). MiR200s expression was normalized to the expression of U6. All qRT-PCR reactions were performed in duplicate on a StepOnePlus RT-PCR system (Applied Biosystems). The relative mRNA and miRNA expression levels were calculated by $2^{-\Delta \Delta \mathrm{Ct}}$ method. Primer sequences are shown in Table 2.

\section{Protein extraction and Western Blot}

For the extraction of total protein, cells were lysed in radio-immunoprecipitation assay (RIPA) buffer (Beyotime) containing PMSF (Sigma). The concentration of total protein was quantified using BCA protein assay kit (Beyotime). Equal amounts $(60 \mu \mathrm{g})$ of proteins were separated by $10 \%$ SDSPAGE and transferred onto PVDF membranes $(0.45 \mu \mathrm{m}$; Millipore). The membranes were blocked with 5\% fat-free milk in Tris-buffered saline containing 0.1\% Tween-20 (TBST) for $1 \mathrm{~h}$. Subsequently, the blots were incubated overnight at $4^{\circ} \mathrm{C}$ with E-cadherin $(1: 1000 ;$ Abcam), Vimentin (1:1000; Affinity, USA), Zeb1 (1:250; Affinity), Zeb2 (1:250; Affinity) and GAPDH (1:4000; Proteintech, USA). After washing three times with TBST and incubating with secondary antibody (anti-rabbit antibody, 1:4000; Affinity) at room temperature for $1 \mathrm{~h}$, the membranes were washed again and incubated with enhanced ECL detection reagent (Millipore). Finally, the intensities of protein bands were quantified using ImageJ, version $1.5(\mathrm{NIH})$.

\section{Transwell migration and invasion assays}

$8.0 \mu \mathrm{m}$ diameter filters (Corning Costar, USA) were used for migration and invasion assays. For invasion assay, working matrigel (1:3 diluted with FBS-free DMEM/F12 or 1640 medium) (Becton Dickinson and Company) was used on the upper chamber of the filters. EECs were resuspended in phenol red-free DMEM/F12 with serum-free FBS and then loaded into the upper chamber, while phenol redfree DMEM/F12 with $40 \%$ FBS was loaded into the lower chamber. Ishikawa cells were resuspended in phenol red-free 1640 culture medium with serum-free FBS. And phenol redfree 1640 medium with $10 \%$ FBS was added to the lower chamber. $E_{2}$ and/or PHTPP were added in the resuspended cells using working dose for hormone treatment. After 24-h incubation, the cells that migrated to the lower chamber were evaluated for migration. After 48-h incubation, the cells that invaded the lower chamber were evaluated for invasion. Matrigel and non-invaded cells on the upper side of filters were removed with cotton swabs. Finally, the remaining cells were fixed and stained with $0.1 \%$ crystal violet prior to microscopic visualization at $\times 200$ magnification. All experiments were performed in triplicate wells for each condition, and independently repeated three times.

Table 2 Primers used in qRT-PCR.

\begin{tabular}{|c|c|c|}
\hline Gene & Forward primer & Reverse primer \\
\hline MALAT1 & 5'-GCСАСТTCTCAACCGTCCCT-3' & 5'-AACACCTCACAAAACCCCCG-3' \\
\hline Zeb1 & 5'-ATCATCGCTACTCСТACTG-3' & 5'-TCTTCССТTGTCAAACTC-3' \\
\hline Zeb2 & 5'-TGACAAAATGATTGAGAACC-3' & 5'-TACAGATGACAAGAGGAGGG-3' \\
\hline E-cadherin & 5'-ATTTTTCCCTCGACACCCGAT-3' & $5^{\prime}$ - TCCCAGGCGTAGACCAAGA-3' \\
\hline Vimentin & 5'-AGTCCACTGAGTACCGGAGAC-3' & 5'- CATTTCACGCATCTGGCGTTC-3' \\
\hline GAPDH & 5'-TCAGGCGTCTGTAGAGGCTT-3' & 5'- ATGCACATCCTTCGATAAGACTG-3' \\
\hline has-miR200a-3p & 5'-TGCTAACACTGTCTGGTAACGATGT-3' & \\
\hline has-miR200b-3p & 5'-CGTAATACTGCCTGGTAATGATGA-3' & \\
\hline has-miR200c-3p & 5'-ATACTGCCGGGTAATGATGGA-3' & \\
\hline has-miR141-3p & 5'-TGCTAACACTGTCTGGTAAAGATGG-3' & \\
\hline has-miR429 & 5'-TGGTAATACTGTCTGGTAAAACCGT-3' & \\
\hline U6 & 5'-CTCGCTTCGGCAGCACA-3' & 5'-AACGCTTCACGAATTTGCGT-3' \\
\hline
\end{tabular}

The reverse primer of miRNAs is the Universal Reverse Primer (RiboBio, Guangzhou). 


\section{Statistical analysis}

All statistical analyses were performed using GraphPad Prism 6 (GraphPad Software Inc.). Student's t-test and ANOVA were used to compare the differences between two groups with a normal distribution of variables. For the comparison of nonnormally distributed variables, the Kruskal-Wallis test was applied. The data were expressed as mean \pm S.D. $P$ values of less than 0.05 were considered statistically significant $\left({ }^{*} P<0.05\right.$, $* * P<0.01$ and $* * * P<0.001)$.

\section{Results}

\section{Expression levels of MiR200s and MALAT1 in endometriosis patients}

The expression of miR200s, MALAT1, Zeb1, Zeb2, E-cadherin and Vimentin were examined in 20 ectopic endometrium (ovarian endometriosis samples), 20 eutopic endometrium and 20 normal endometrium by qRT-PCR detection. Compared to normal and eutopic groups, a significantly decreased expression of miR200s was found in ovarian endometriosis samples (Fig. 1A). There was no significant difference between eutopic group and normal group. MALAT1 expression was
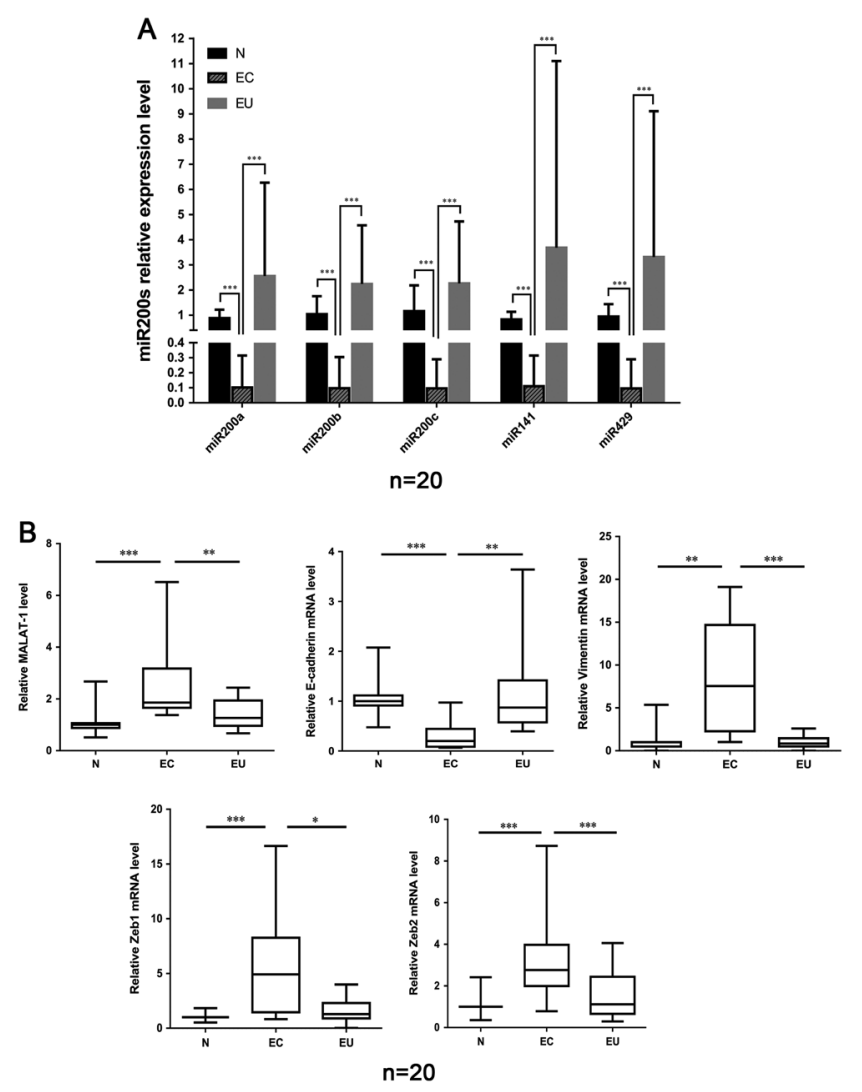

Figure 1 (A) Expression level of miR200s in normal (N), ectopic (ovarian) (EC) and eutopic (EU) endometriosis. (B) Expression levels of MALAT1, Zeb1, Zeb2, E-cadherin and Vimentin in normal, ectopic (ovarian) and eutopic endometriosis examined by qRT-PCR. (*:

$P<0.05$; ${ }^{* *}: P<0.01 ;{ }^{* * *} P<0.001$ by the Kruskal-Wallis test.) upregulated in ectopic tissues compared to normal and eutopic endometrium groups (Fig. 1B). Moreover, the increased expression levels of Zeb1, Zeb2 and Vimentin, and decreased E-cadherin expression were observed in ectopic group, indicating that EMT is involved in the progression of endometriosis (Fig. 1B). No significant differences were noted in the expression levels of MALAT1, Zeb1, Zeb2, E-cadherin and Vimentin between normal and eutopic groups.

\section{$E_{2}$ regulates miR200s and MALAT1 and promotes EMT in EECs}

ERE was detected in the promoter region of MALAT1 via JASPAR, suggesting a relationship between estrogen and MALAT1 (Supplementary Fig. 1, see section on supplementary data given at the end of this article). Hence, $17 \beta$-Estradiol $\left(E_{2}\right)$ was used to explore the association among estrogen, EMT and miR200s. At first, EECs were isolated and preliminary characterized. EECs were grown in clusters, displayed typical cobblestone morphology and exceeded a high population purity of over 95\% (Supplementary Fig. 2).

To determine the relationship between $E_{2}$ and EMT in endometriosis, EECs were treated with different concentrations $\left(0,10^{-12}, 10^{-10}, 10^{-8}, 10^{-6} \mathrm{~mol} / \mathrm{L}\right)$ of $\mathrm{E}_{2}$ for $48 \mathrm{~h}$ (Fig. 2A). Subsequently, the protein expression levels of Zeb1, Zeb2, E-cadherin and Vimentin were detected by Western blotting. Zeb1 and Zeb2 proteins reached maximum expression at $10^{-8} \mathrm{~mol} / \mathrm{L}$. $\mathrm{E}_{2}$ upregulated Vimentin expression and downregulated E-cadherin expression in a dose-dependent manner. EECs were also treated with $\mathrm{E}_{2}$ at $10^{-8} \mathrm{~mol} / \mathrm{L}$ for $0,24,48$ or $72 \mathrm{~h}$ to assess the effect of time on expression variance (Fig. 2B). Protein expression levels of Zeb1 and Zeb2 were statistically upregulated by $E_{2}$ treatment starting from $48 \mathrm{~h}$. E-cadherin expression was downregulated at $48 \mathrm{~h}$ and $72 \mathrm{~h}$. Vimentin expression was upregulated at $24 \mathrm{~h}$ also in a time-dependent fashion.

The RNA expression levels in EECs treated with different concentrations $\left(0,10^{-12}, 10^{-10}, 10^{-8}\right.$ and $\left.10^{-6} \mathrm{~mol} / \mathrm{L}\right)$ of $\mathrm{E}_{2}$ for $24 \mathrm{~h}$ were analyzed by qRT-PCR method. The results showed that all members of miR200s were downregulated from $10^{-8} \mathrm{~mol} / \mathrm{L}$, while MALAT1 expression was upregulated (Fig. 2C and D). Both were dose dependent. Therefore, EECs treated with $10^{-8} \mathrm{~mol} / \mathrm{L}$ of $E_{2}$ for $48 \mathrm{~h}$ were used in the subsequent analysis.

\section{ERß may participate in miR200s-induced EMT in EECs and Ishikawa cells}

To confirm the effects of $E_{2}$ on EMT in endometriosis, EECs were treated with $10^{-6} \mathrm{~mol} / \mathrm{L}$ of $\mathrm{ER} \alpha$ antagonist $\mathrm{ICl}$. However, the effects of $\mathrm{E}_{2}$ on miR200s, Zeb1 and Zeb2 were not inhibited by ICI (Supplementary Fig. 3). Therefore, PHTPP $\left(10^{-5} \mathrm{~mol} / \mathrm{L}\right)$, an ER $\beta$ antagonist, was used to inhibit the effect of $E_{2}$ on miR200s, Zeb1 and 
A
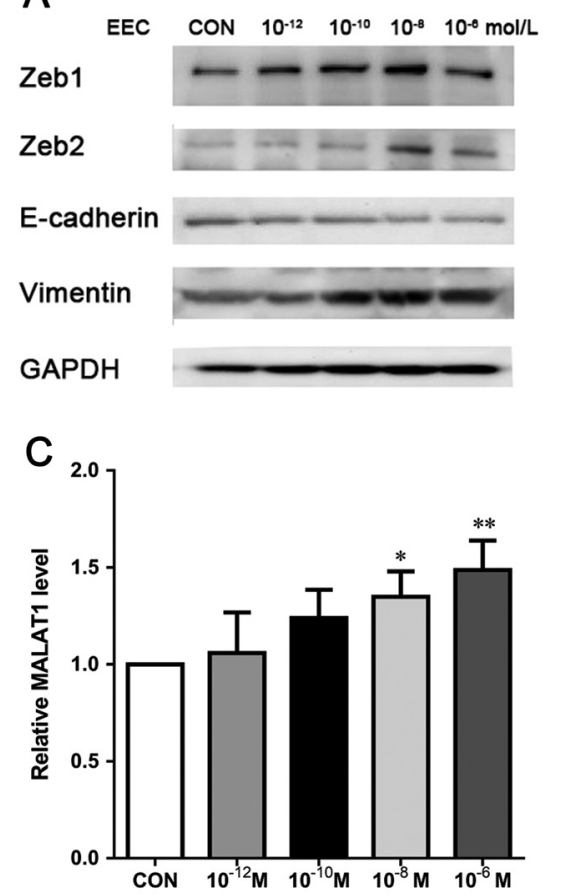

B

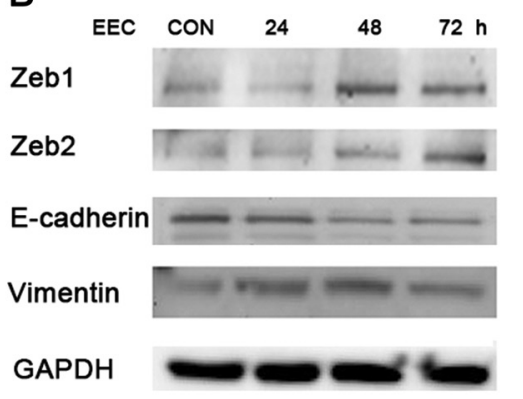

D

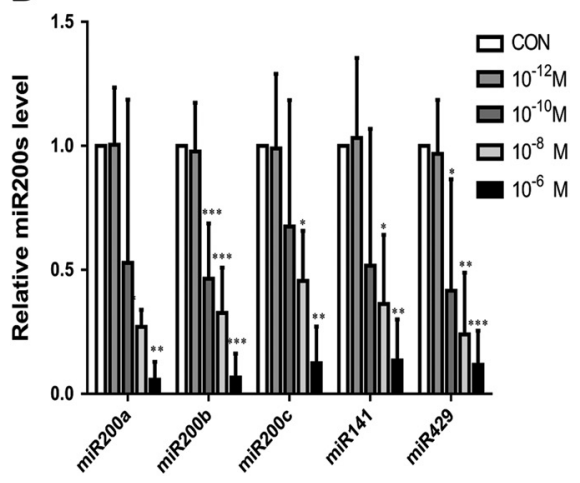

Figure 2 ( $A$ and B) Expression levels of EMT-associated markers (Zeb1, Zeb2,

E-cadherin and Vimentin) with $E_{2}$ treatment for different doses $\left(0,10^{-12}, 10^{-10}, 10^{-8}\right.$, $\left.10^{-6} \mathrm{~mol} / \mathrm{L}\right)$ and time $(0,24,48$ or $72 \mathrm{~h})$ in EECs detected by Western blot.(M: mol/L); (C and D) Expression levels of MALAT1 and miR200s under $E_{2}$ for various doses $\left(0,10^{-12}\right.$, $10^{-10}, 10^{-8}, 10^{-6} \mathrm{~mol} / \mathrm{L}$ ) for $24 \mathrm{~h}$ in EECs measured by qRT-PCR. Data were evaluated by one-way ANOVA analysis $\left({ }^{*} P<0.05\right.$, ${ }^{* *} P<0.01,{ }^{* * *} P<0.001$ compared with untreated group).
Zeb2 in EECs. The results of Western blot and qRT-PCR demonstrated that $E_{2}$-inducted expression levels of Zeb1, Zeb2, E-cadherin and Vimentin were antagonized by PHTPP in both protein and mRNA levels (Fig. 3A and $B)$. The results of qRT-PCR indicated that PHTPP could reverse $E_{2}$-inhibited miR200s and $E_{2}$-induced MALAT1 expression (Fig. 3C and D). Furthermore, EECs were stimulated with $E_{2}$ and/or PHTPP to determine the role of $\mathrm{E}_{2}$ in cell migration and invasion. As shown in Fig. 3E, $E_{2}$ significantly increased the migration and invasion of EECs. By contrast, $E_{2}$-mediated migration and invasion were inhibited by PHTPP.

In the subsequent studies, Ishikawa cells were used as a substitute for EECs. Hence, the aforementioned experiments were repeated on Ishikawa cells, and similar results were obtained (Supplementary Fig. 4).

\section{MiR200c inhibits MALAT1 expression and reverses $E_{2^{-}}$ mediated EMT}

To examine the correlation of MALAT1 and miR200s, RNAhybrid (https://bibiserv.cebitec.uni-bielefeld.de/ rnahybrid) was used to predict the potential interaction between them. A negative value of MFE (minimum free energy) can indicate the degree of interaction between miRNA and target mRNA/IncRNA. A greater absolute value of MFE denotes a more reliable bond. The results of RNAhybrid prediction indicated a strong bonding strength between miR200c and MALAT1 (Fig. 4A).

To examine the relationship among $\mathrm{E}_{2}$, miR200c and MALAT1, Ishikawa cells were transiently transfected with miR200c mimic. Overexpression of miR200c in miR200c mimic-transfected Ishikawa cells was inhibited by $10^{-8} \mathrm{~mol} / \mathrm{L}$ of $\mathrm{E}_{2}$ treatment (Fig. $4 \mathrm{~B}$ ). Besides, the overexpression of miR200c resulted in a decrease in MALAT1 expression (Fig. 4C). Similarly, $E_{2}$ reversed the miR200c-inhibited MALAT1 expression. Western blot analysis revealed that miR200c inhibited the protein expression levels of Zeb1, Zeb2 and Vimentin, as well as upregulated E-cadherin protein expression with or without $\mathrm{E}_{2}$ treatment (Fig. 4D). Additionally, $\mathrm{E}_{2}$ reduced the effect of miR200c on EMT-associated markers. These results suggest that miR200c reverses $E_{2}$-mediated EMT in Ishikawa cells. Furthermore, Transwell assay revealed that miR200c inhibited the migration and invasion of Ishikawa cells with or without $\mathrm{E}_{2}$ treatment (Fig. 4E). Taken together, this study demonstrates that miR200c reverses $\mathrm{E}_{2}$-mediated EMT and attenuates the expression of MALAT1.

\section{Knockdown of MALAT1 increases miR200s and attenuates $E_{2}$-induced $E M T$}

To elucidate the role of MALAT1 in endometriosis, the expression of MALAT1 was inhibited to observeexpression of miR200s, Zeb1, Zeb2, E-cadherin and Vimentin, and the processes of migration and invasion. Compared to negative control cells treated with siRNA (si-NC), two MALAT1-specific siRNAs, namely si-MALAT1-1 and si-MALAT1-2, were specifically designed to suppress MALAT1 expression in Ishikawa cells for $24 \mathrm{~h}$ (Fig. 5A). The expression of MALAT1 was detected using qRT-PCR. 

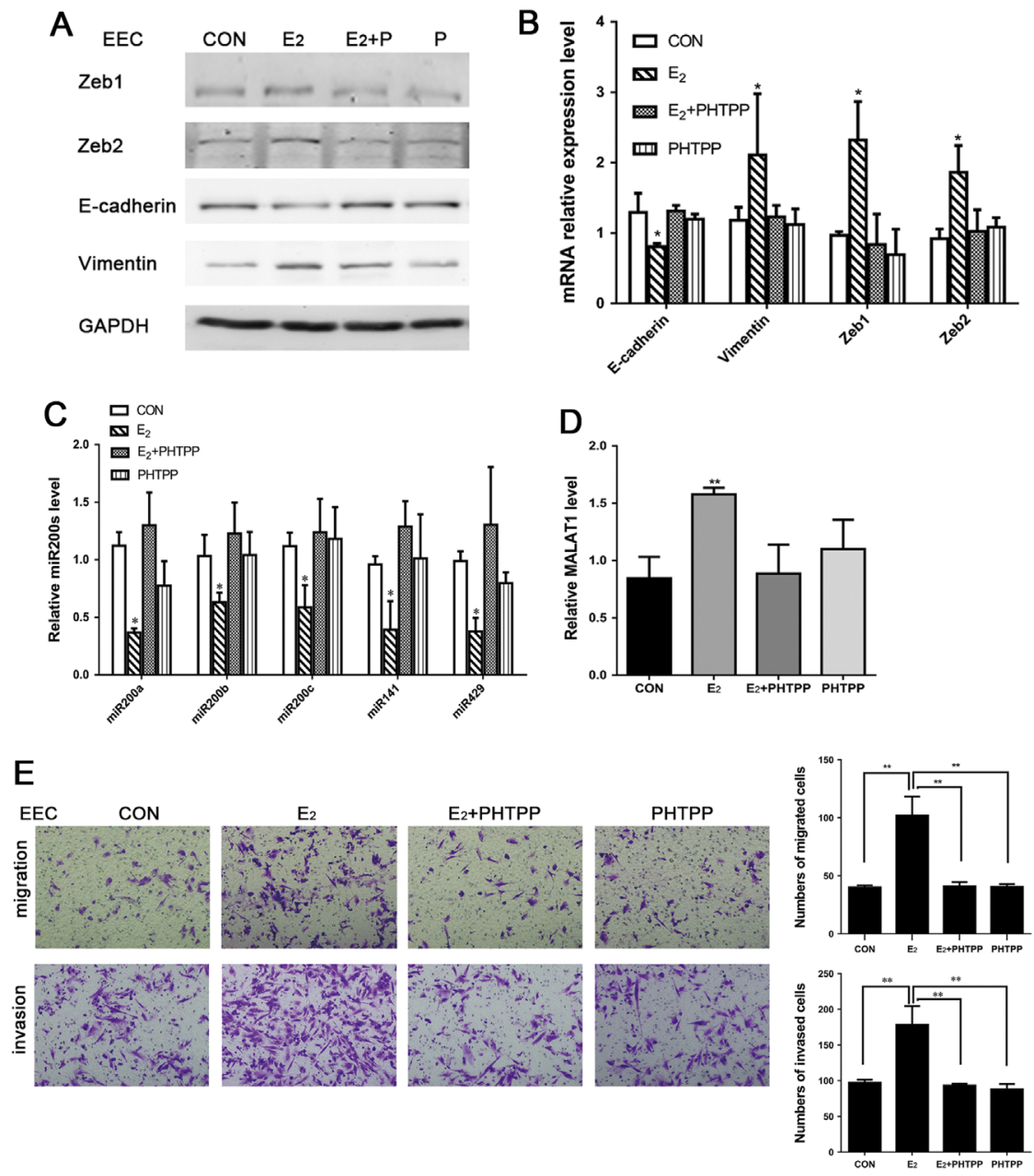

Figure 3 (A) Protein expression levels of EMT-associated markers in EECs with treatment of $E_{2}$ and/or PHTPP for $48 \mathrm{~h}$ analyzed by Western blot; (B, C and D) RNA expression levels of EMT-associated markers, MALAT1 and miR200s treated with $E_{2}$ and/or PHTPP in EECs for $24 \mathrm{~h}$ analyzed by qRT-PCR; (E) $E_{2}$ promoted migration and invasion of EECs and PHTPP reversed the effect. Data were evaluated by one-way ANOVA analysis $\left({ }^{*} P<0.05,{ }^{* *} P<0.01,{ }^{* * *} P<0.001\right)$.

Photographs were taken at magnifications of $200 \times$.

Notably, si-MALAT1-2 greatly reduced the expression of MALAT1 and was selected for cell transfection in the subsequent study. $\mathrm{E}_{2}\left(10^{-8} \mathrm{~mol} / \mathrm{L}\right)$ was added to examine whether MALAT1 can cause a great difference to $E_{2}$-mediated EMT in endometriosis. The inhibition of MALAT1 by si-MALAT1-2 in cells was reversed by the addition of $E_{2}$ (Fig. 5B). After 24-h incubation with $E_{2}$, the expression levels of MALAT1 were both upregulated in cells transfected with si-NC and si-MALAT1, while the effect of RNA interference on reduced MALAT1 expression was indeed observed. Besides, transfection with si-MALAT1-2 for $24 \mathrm{~h}$ increased the expression of miR200s (Fig. 5C). MALAT1 knockdown decreased the expression levels of Zeb1, Zeb2 and Vimentin protein and increased E-cadherin protein levels at $48 \mathrm{~h}$ (Fig. 5D). Following $E_{2}$ treatment, the changes in expression levels of Zeb1, Zeb2, E-cadherin and Vimentin were affected by MALAT1 inhibition. In sum, a positive correlation was found between MALAT1 and EMT-associated biomarkers in endometriosis. Furthermore, Transwell migration and invasion assays were used to support the role of MALAT1 in $E_{2}$-induced EMT. Specifically, the migration and invasion abilities of MALAT1-knockdown cells were suppressed after $E_{2}$ treatment (Fig. 5E).
These results suggest that MALAT1 is involved in $\mathrm{E}_{2}$-induced cell migration and invasion. Knockdown of MALAT1, at least to some extent, can reverse $\mathrm{E}_{2}$-mediated EMT in Ishikawa cells.

\section{Reciprocal inhibition between miR200s and MALAT1 affects EMT}

Previous experiments revealed the effects of miR200s and MALAT1 on $E_{2}$-mediated EMT. In this experimental section, the reciprocal inhibition between miR200s and MALAT1 were re-examined by using miR200c inhibitor and si-MALAT1. MiRNA inhibitors competitively bind to miRNAs through sequence complementarity principle to suppress the inhibitory effect of miRNAs on their respective target mRNAs. MiRNAs themselves might not be degraded, and their expression levels should theoretically remain unchanged. In other words, the detection of miR200s by qRT-PCR method is meaningless. To verify the effect of miR200c inhibitor, Western blotting was used to detect the expression level of EMT-associated markers and EMT transcription factors. The inhibition of miR200c contributed to the upregulation of Zeb1, Zeb2 and Vimentin, and 


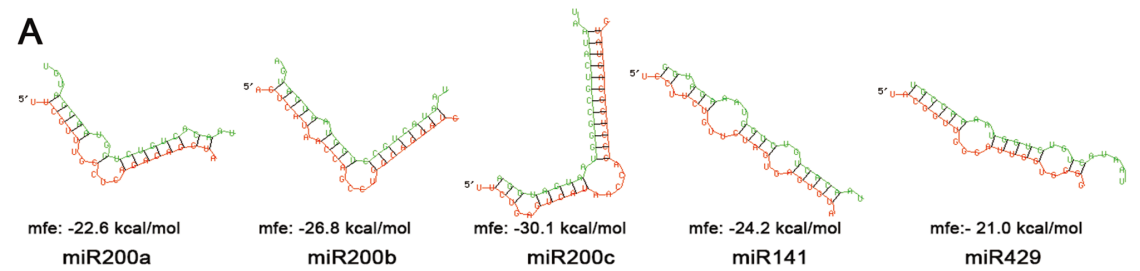

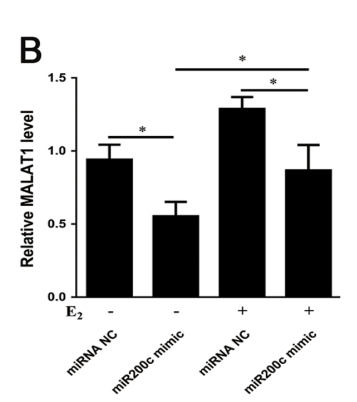
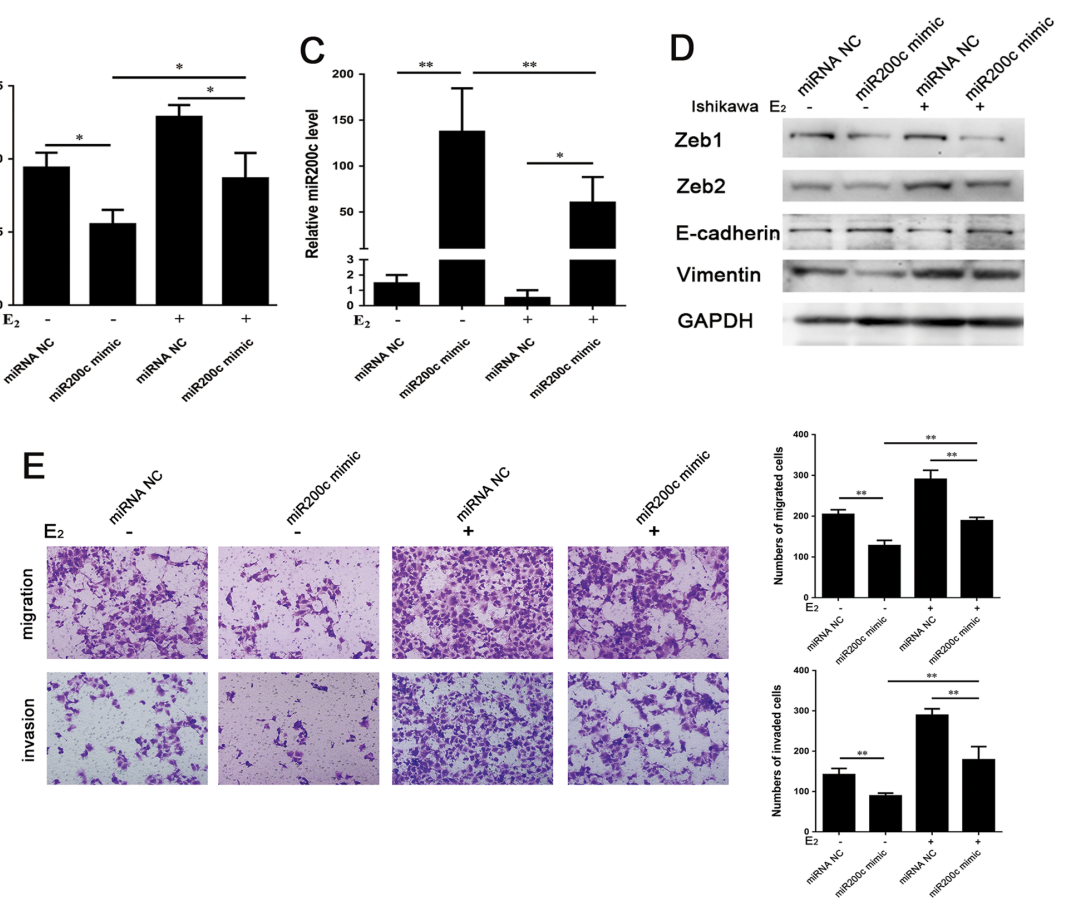

Figure 4 (A) The predicted results of the interaction between MALAT1 and miR200s by RNAhybrid (https://bibiserv.cebitec.unibielefeld.de/rnahybrid). (Red: MALAT1. Green: each member of miR200s) (MALAT1 and miR200a, $\mathrm{mfe}:-22.6 \mathrm{kcal} / \mathrm{mol}$; MALAT1 and miR200b, mfe: $-26.8 \mathrm{kcal} / \mathrm{mol}$; MALAT1 and miR200c, mfe: $-30.1 \mathrm{kcal} / \mathrm{mol}$; MALAT1 and miR141, mfe: $-24.2 \mathrm{kcal} / \mathrm{mol}$; MALAT1 and miR429, mfe: $-21.0 \mathrm{kcal} / \mathrm{mol}) ;.(B$ and $\mathrm{C}$ ) Ishikawa cells were transfected with miR200c mimic or miRNA Negative Control (miRNA $\mathrm{NC})$ and were treated with or without $\mathrm{E}_{2}$ $\left(10^{-8} \mathrm{~mol} / \mathrm{L}\right)$ for $24 \mathrm{~h}$. The expression levels of miR200c and MALAT1 were analyzed by qRT-PCR. (D) Expression levels of EMTassociated markers detected by Western blot after the same treatment for $48 \mathrm{~h}$. (E) The function of miR200c mimic and/or $\mathrm{E}_{2}$ affects the ability of migration $(24 \mathrm{~h})$ and invasion $(48 \mathrm{~h})$ in Ishikawa cells. Data were evaluated by one-way ANOVA analysis $\left({ }^{*} P<0.05\right.$, $\left.{ }^{* *} P<0.01,{ }^{* * *} P<0.001\right)$. Photographs were taken at magnifications of $200 \times$.
E-cadherin down-regulation at $48 \mathrm{~h}$ (Fig. 6A). Next, Ishikawa cells were treated with miR200c inhibitor after transfection with si-MALAT1. Even though Zeb1, Zeb2 and Vimentin were downregulated by si-MALAT1, their expression levels were increased by the addition of miR200c inhibitor (Fig. 6B). Additionally, the overexpression of Zeb1, Zeb2 and Vimentin induced by the miR200c inhibitor could be attenuated via MALAT1 inhibition. The antagonism effect between miR200c and MALAT1 was also reflected by the expression of E-cadherin. Likewise, the inhibition of EMT via si-MALAT1 could be enhanced by miR200c inhibitor (Fig. 6C). The mutual inhibitory effects of miR200s and MALAT1 on EMT were further supported by the data of Transwell migration and invasion assays (Fig. 6D).

\section{Discussion}

In the present study, our results indicated lower expression levels of epithelial markers (miR200s and E-cadherin) and increased expression levels of mesenchymal markers (MALAT1, Zeb1, Zeb2 and Vimentin) in ovarian endometriosis samples, suggesting that EMT is involved in endometriosis. Bioinformatics analysis was used to predict the existence of ERE on MALAT1 promoter region and the potential interaction between
MALAT1 and miR200s. $E_{2}$ and PHTPP treatments were used to reveal their effects on EMT induction, as well as miR200s and MALAT1 expression. Overexpression of miR200c inhibited MALAT1 expression and EMT progression. Conversely, RNAi-mediated knockdown of MALAT1 prevented EMT and increased miR200s expression. However, this trend could be reversed by $E_{2}$ treatment. Eventually, the inhibition of MALAT1 on EMT was reversed after the suppression of miR200c via miR200c inhibitor.

Numerous IncRNAs have emerged as significant regulators in the development of diseases. MALAT1, which is often highly expressed in various types of cancers cells, has been reported to be associated with proliferation, metastasis and apoptosis (Yoshimoto et al. 2016). Research has shown that MALAT1 promotes the development and progression of cancers via EMT induction. In addition, its expression is regulated by $E_{2}$ in osteosarcoma, prostate and breast cancer cells (Aiello et al. 2016, Hu et al. 2017). In this study, the results of JASPAR prediction indicated the presence of ERE in MALAT1 promoter, which could account for the binding of $E_{2}$ to $E R \alpha$ and $E R \beta$. ER $\alpha$ is primarily expressed in the uterus and is crucial for the development of endometrial tissues (Matthews \& Gustafsson 2003). ER $\beta$, on the other hand, is primarily expressed in the ovary 

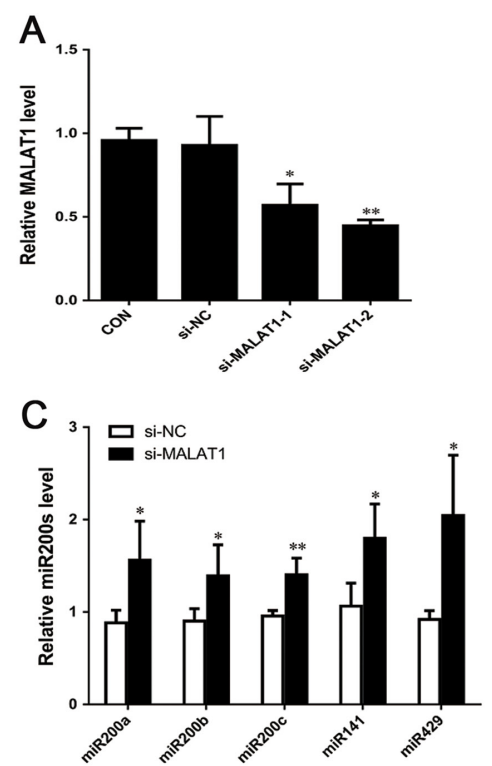

E
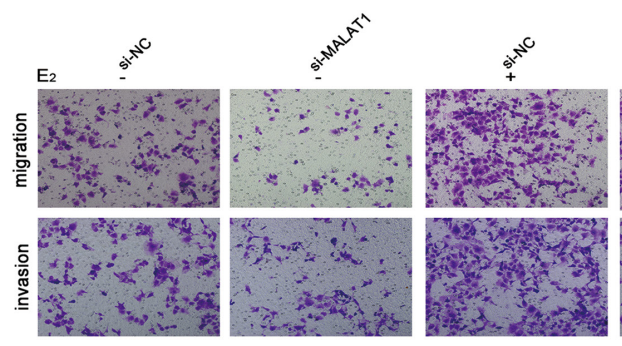
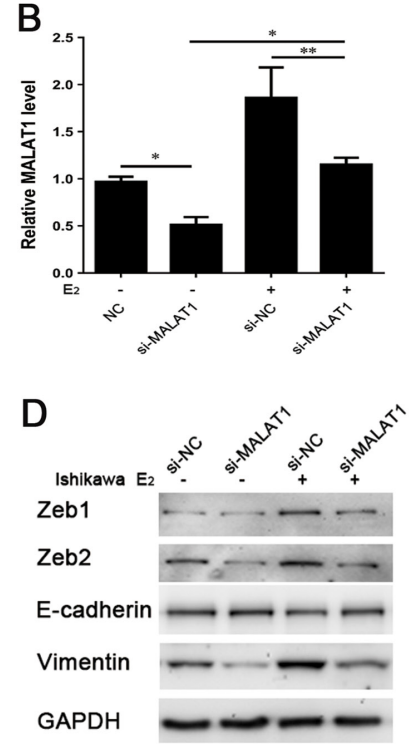

D
Figure 5 (A) Ishikawa cells were transfected with MALAT1 siRNA (si-MALAT1-1 and si-MALAT1-2), expression level of MALAT1 was quantified by qRT-PCR. (B) Ishikawa cells were transfected with si-MALAT1(-2) with or without addition of $E_{2}\left(10^{-8} \mathrm{~mol} / \mathrm{L}\right)$ for $24 \mathrm{~h}$, expression of MALAT1 was quantified by qRT-PCR; (C) Ishikawa cells were transfected with si-MALAT1-2 with or without the addition of $\mathrm{E}_{2}\left(10^{-8} \mathrm{~mol} / \mathrm{L}\right)$ for $24 \mathrm{~h}$, expression levels of miR200s were quantified by qRT-PCR; (D) The altered expression levels of EMT-associated markers after the same treatment for $48 \mathrm{~h}$ were analyzed by Western blot. (E) the function of si-MALAT1(-2) and/or $E_{2}$ affects the ability of migration ( $\left.24 \mathrm{~h}\right)$ and invasion (48h) in Ishikawa cells. Data were evaluated by one-way ANOVA analysis and Student's $t$-test $\left({ }^{*} P<0.05,{ }^{* *} P<0.01\right.$,

$* * * P<0.001)$. Photographs were taken at magnifications of $200 \times$. and has been reported to enhance the invasion ability of endometrial tissues and promote the establishment of ovarian endometrial foci (Han et al. 2015). There are thus a number of good reasons to believe that MALAT1 may interact with $\mathrm{E}_{2}$ and contribute to the development of endometriosis through EMT induction.

LncRNAs are able to post-transcriptionally regulate gene expression by competitively binding to miRNAs. A recent study has demonstrated elevated MALAT1 expression and decreased miR200c expression in endometriosis and suggested that MALAT1/miR200c may be involved in regulating EMT during the development of endometriosis (Liang et al. 2017). Through RNAhybrid, miR200c was predicted to exhibit the strongest interaction with MALAT1. At present, the use of primary endometrial stromal cells to reveal the process of EMT remains less convictive. Instead, EECs, Ishikawa cells and $12 \mathrm{Z}$ cell lines are often used to illuminate the mechanisms of EMT in endometriosis (Chen et al. 2010, Eggers et al. 2016, Xiong et al. 2016). Additionally, the causes of the change in MALAT1 and miR200c are still unexplained at molecular level. With endometriosis being an estrogen-dependent disease and the existence of ERE in MALAT1 promoter, there are reasons to believe that estrogen plays a key role in MALAT1/miR200c-associated endometriosis. Therefore, we are committed to remedy the deficiencies and uncover the root cause of the expression changes.

$E_{2}$ acts as a crucial player in the development and progression of endometriosis (Zhang et al. 2016). $E_{2}$ upregulated Zeb1, Zeb2 and Vimentin and reduced E-cadherin expression, indicating its role of promoting EMT. Moreover, the expression of miR200s was reduced following $E_{2}$ treatment. The inhibitory effect of $E_{2}$ on miR200s expression was diminished in the presence of ER $\beta$ antagonist PHTPP. The expression of MYC is significantly upregulated by upregulated by estrogen in ectopic endometrium and associated with cell proliferation in endometriosis (Proestling et al. 2015). MYC-regulated miRNAs, such as miR200a, miR200b, miR106a and miR153a-1, contain ERß-binding sites, and their expression levels can be adjusted by both MYC and ER $\beta$ (Edvardsson et al. 2013). Similarly, other studies have indicated that the suppression of miR200a/b is a 
A

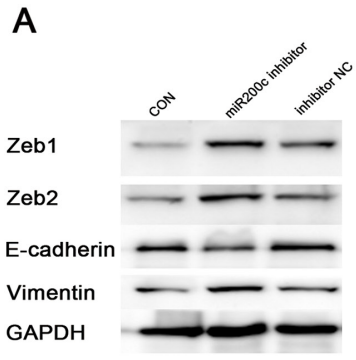

B si-No

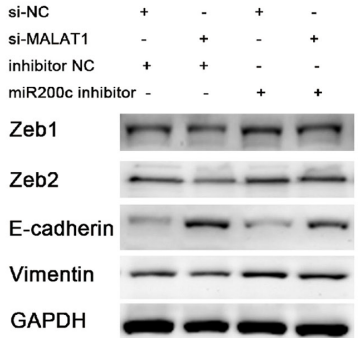

C

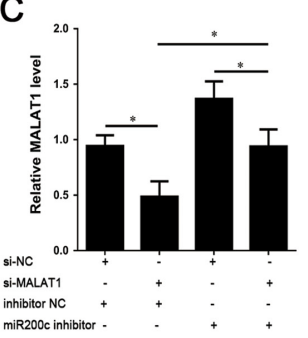

D

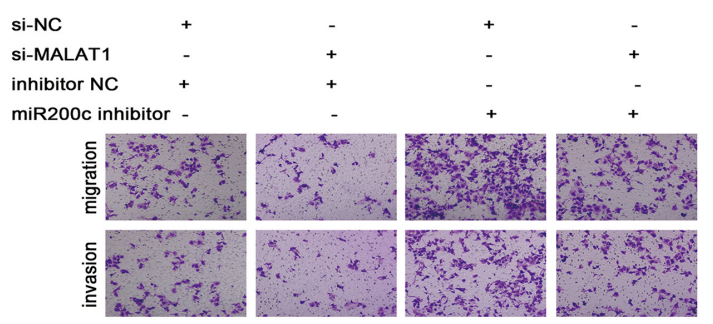

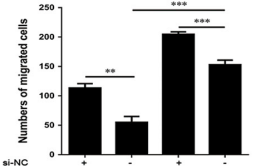

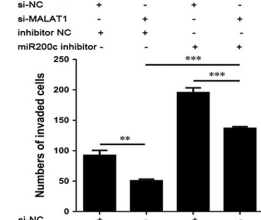

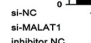

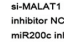

$E$

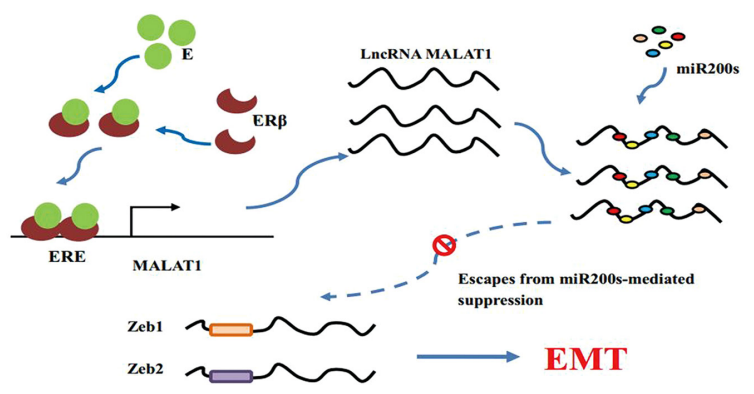

consequence of ER $\beta$ expression in colon cancer cells. Despite the controversy, more studies suggest that ER $\beta$ is highly expressed in ectopic lesions compared to normal endometrial tissue (Bulun et al. 2012, Izawa et al. 2016). Such evidence may explain the reverse effect of $\mathrm{E}_{2}$-inhibited miR200s by PHTPP. The effect of PHTPP on reversing $E_{2^{-}}$ induced MALAT1 expression was also groundbreaking. It may be the secondary change following the alteration of miR200s expression, and the $\mathrm{E}_{2}$-regulated ER $\beta$ activation due to the presence of ERE in MALAT1 promoter. Indeed, ERo-mediated MALAT1 expression changes cannot be ruled out. Further research is required to explore the molecular mechanism of $E_{2}$ on MALAT1 expression.

Nevertheless, there are few unavoidable limitations in this study. First, although Ishikawa cells have been demonstrated to exert the same effect as EECs in response to $E_{2}$ treatment, these cells might possess certain fundamental deficiencies. Thus, the establishment of more convincing immortalized endometriotic cell lines is necessary in the future studies of endometriosis. Besides, this study only focused on the role of $E_{2}$-mediated EMT in endometriosis via MALAT1/miR200s axis. Hence, more research is needed to verify the complex relationship among ER $\beta$, MALAT1 and miR200s and unravel the molecular mechanisms underlying their interactive effects.

In summary, our results demonstrate $E_{2}$ can promote EMT in endometriosis by altering miR200s and MALAT1 expression levels. A significant reciprocal inhibition is identified between miR200s and MALAT1. $\mathrm{E}_{2}$-mediated MALAT1/miR200s expression may affect cell migration and invasion in endometriosis, by regulating EMT. These findings not only improve our understanding of the mechanisms underlying EMT induction in endometriosis, but also provide evidence to support $\mathrm{E}_{2}$-mediated MALAT1/ miR200s axis as a new therapeutic target for endometriosis.

\section{Supplementary data}

This is linked to the online version of the paper at https://doi.org/10.1530/REP-18-0424.

\section{Declaration of interest}

The authors declare that there is no conflict of interest that could be perceived as prejudicing the impartiality of the research reported.

\section{Funding}

This project was supported by the National Natural Science Foundation of China (Grant No. 81471439 to Yi Liu). 


\section{Author contribution statement}

$Y L$ and $L Z$ designed and performed analyses. $Y D, H L$ and $Z Z$ participated in the study design, analysis and manuscript drafting. $W X$ and $L Z$ helped in the research design. $Y D, H L$, $\mathrm{N} \mathrm{L}, \mathrm{H} \mathrm{H}$ and $\mathrm{Q} \mathrm{L}$ performed the experiments. All authors have approved the final manuscript.

\section{References}

Aiello A, Bacci L, Re A, Ripoli C, Pierconti F, Pinto F, Masetti R, Grassi C, Gaetano C, Bassi PF et al. 2016 MALAT1 and HOTAIR long noncoding RNAs play opposite role in estrogen-mediated transcriptional regulation in prostate cancer cells. Scientific Reports 6 38414. (https:// doi.org/10.1038/srep38414)

Bulun SE, Monsavais D, Pavone ME, Dyson M, Xue Q, Attar E, Tokunaga H \& Su EJ 2012 Role of estrogen receptor-beta in endometriosis. Seminars in Reproductive Medicine 30 39-45. (https://doi. org/10.1055/s-0031-1299596)

Chen Y-J, Li H-Y, Huang C-H, Twu N-F, Yen M-S, Wang P-H, Chou T-Y, Liu Y-N, Chao K-C \& Yang M-H 2010 Oestrogen-induced epithelialmesenchymal transition of endometrial epithelial cells contributes to the development of adenomyosis. Journal of Pathology 222 261-270. (https://doi.org/10.1002/path.2761)

Crosignani P, Olive D, Bergqvist A \& Luciano A 2006 Advances in the management of endometriosis: an update for clinicians. Human Reproduction Update 12 179-189. (https://doi.org/10.1093/humupd/ dmi049)

Dhamija S \& Diederichs S 2016 From junk to master regulators of invasion: IncRNA functions in migration, EMT and metastasis. International Journal of Cancer 139 269-280. (https://doi.org/10.1002/ijc.30039)

Edvardsson K, Nguyen-Vu T, Kalasekar SM, Ponten F, Gustafsson J-A \& Williams C 2013 Estrogen receptor beta expression induces changes in the microRNA pool in human colon cancer cells. Carcinogenesis $\mathbf{3 4}$ 1431-1441. (https://doi.org/10.1093/carcin/bgt067)

Eggers JC, Martino V, Reinbold R, Schafer SD, Kiesel L, Starzinski-Powitz A, Schuring AN, Kemper B, Greve B \& Gotte M 2016 MicroRNA miR-200b affects proliferation, invasiveness and stemness of endometriotic cells by targeting ZEB1, ZEB2 and KLF4. Reproductive Biomedicine Online 32 434-445. (https://doi.org/10.1016/j.rbmo.2015.12.013)

Feng X, Wang Z, Fillmore R \& Xi Y 2014 MiR-200, a new star miRNA in human cancer. Cancer Letters 344 166-173. (https://doi.org/10.1016/j. canlet.2013.11.004)

Giudice LC \& Kao LC 2004 Endometriosis. Lancet 364 1789-1799. (https:// doi.org/10.1016/S0140-6736(04)17403-5)

Han SJ, Jung SY, Wu S-P, Hawkins SM, Park MJ, Kyo S, Qin J, Lydon JP, Tsai SY, Tsai M-J et al. 2015 Estrogen receptor beta modulates apoptosis complexes and the inflammasome to drive the pathogenesis of endometriosis. Cell 163 960-974. (https://doi.org/10.1016/j. cell.2015.10.034)

Hawkins SM, Creighton CJ, Han DY, Zariff A, Anderson ML, Gunaratne PH \& Matzuk MM 2011 Functional microRNA involved in endometriosis. Molecular Endocrinology 25 821-832. (https://doi.org/10.1210/ me.2010-0371)

Hu Q, Li S, Chen C, Zhu M, Chen Y \& Zhao Z2017 17 Beta-estradiol treatment drives Sp1 to upregulate MALAT-1 expression and epigenetically affects physiological processes in U2OS cells. Molecular Medicine Reports 15 1335-1342. (https://doi.org/10.3892/mmr.2017.6115)

Huhtinen K, Stahle M, Perheentupa A \& Poutanen M 2012 Estrogen biosynthesis and signaling in endometriosis. Molecular and Cellular Endocrinology 358 146-154. (https://doi.org/10.1016/j. mce.2011.08.022)
Izawa M, Taniguchi F \& Harada T 2016 Molecular background of estrogen receptor gene expression in endometriotic cells. Reproductive Sciences 23 871-876. (https://doi.org/10.1177/1933719115623642)

Kitawaki J, Kado N, Ishihara H, Koshiba H, Kitaoka Y \& Honjo H 2002 Endometriosis: the pathophysiology as an estrogen-dependent disease. Journal of Steroid Biochemistry and Molecular Biology 83 149-155. (https://doi.org/10.1016/S0960-0760(02)00260-1)

Liang Z, Chen Y, Zhao Y, Xu C, Zhang A, Zhang Q, Wang D, He J, Hua W \& Duan P 2017 miR-200c suppresses endometriosis by targeting MALAT1 in vitro and in vivo. Stem Cell Research and Therapy 8 251. (https://doi. org/10.1186/s13287-017-0706-z)

Matthews J \& Gustafsson J-A 2003 Estrogen signaling: a subtle balance between ER alpha and ER beta. Molecular Interventions 3 281-292. (https://doi.org/10.1124/mi.3.5.281)

Park SH, Cheung LW, Wong AS \& Leung PC 2008 Estrogen regulates Snail and Slug in the down-regulation of E-cadherin and induces metastatic potential of ovarian cancer cells through estrogen receptor alpha. Molecular Endocrinology 22 2085-2098. (https://doi.org/10.1210/ me.2007-0512)

Park SJ, Kim JG, Kim ND, Yang K, Shim JW \& Heo K 2016 Estradiol, TGFbeta1 and hypoxia promote breast cancer stemness and EMT-mediated breast cancer migration. Oncology Letters 11 1895-1902. (https://doi. org/10.3892/ol.2016.4115)

Proestling K, Birner P, Gamperl S, Nirtl N, Marton E, Yerlikaya G, Wenzl R, Streubel B \& Husslein H 2015 Enhanced epithelial to mesenchymal transition (EMT) and upregulated MYC in ectopic lesions contribute independently to endometriosis. Reproductive Biology and Endocrinology 13 75. (https://doi.org/10.1186/s12958-015-0063-7)

Sampson JA 1927 Peritoneal endometriosis due to the menstrual dissemination of endometrial tissue into the peritoneal cavity. American Journal of Obstetrics and Gynecology 14 422-469. (https://doi. org/10.1016/S0002-9378(15)30003-X)

Vandewalle C, Van Roy F \& Berx G 2009 The role of the ZEB family of transcription factors in development and disease. Cellular and Molecular Life Sciences 66 773-787. (https://doi.org/10.1007/s00018-008-8465-8)

Xiao H, Tang K, Liu P, Chen K, Hu J, Zeng J, Xiao W, Yu G, Yao W, Zhou H et al. 2015 LncRNA MALAT1 functions as a competing endogenous RNA to regulate ZEB2 expression by sponging miR-200s in clear cell kidney carcinoma. Oncotarget 6 38005-38015. (https://doi.org/10.18632/ oncotarget.5357)

Xiong Y, Liu Y, Xiong W, Zhang L, Liu H, Du Y \& Li N 2016 Hypoxiainducible factor 1alpha-induced epithelial-mesenchymal transition of endometrial epithelial cells may contribute to the development of endometriosis. Human Reproduction 31 1327-1338. (https://doi. org/10.1093/humrep/dew081)

Yang J \& Weinberg RA 2008 Epithelial-mesenchymal transition: at the crossroads of development and tumor metastasis. Developmental Cell 14 818-829. (https://doi.org/10.1016/j.devcel.2008.05.009)

Yoshimoto R, Mayeda A, Yoshida M \& Nakagawa S 2016 MALAT1 long non-coding RNA in cancer. Biochimica et Biophysica Acta 1859 192-199. (https://doi.org/10.1016/j.bbagrm.2015.09.012)

Zhang L, Xiong W, Xiong Y, Liu H \& Liu Y 201617 Beta-estradiol promotes vascular endothelial growth factor expression via the Wnt/beta-catenin pathway during the pathogenesis of endometriosis. Molecular Human Reproduction 22 526-535. (https://doi.org/10.1093/molehr/gaw025)

Received 12 August 2018

First decision 17 September 2018

Revised manuscript received 18 November 2018

Accepted 30 November 2018 